\title{
LV-OP-1-2
}

\section{Salvage living donor liver transplantation versus repeat liver resection for patients with recurrent hepatocellular carcinoma and liver cirrhosis: A propensity score-matched comparison}

\author{
Gi-Won SONG*, Young-In YOON
}

Department of Surgery, Asan Medical Center, Seoul, Korea

Introduction: Following curative liver resection (LR), resectable tumor recurrence in patients with preserved liver function leads to deciding between a repeat LR and a salvage liver transplantation (LT), if a donor liver is available.

Methods: This retrospective study compared survival outcomes following salvage living donor LT (LDLT) and repeat LR in patients with recurrent hepatocellular carcinoma (HCC). We also analyzed the risk factors associated with survival outcomes after salvage LDLT. We reviewed the medical records of patients who underwent repeat LR $(n=163)$ or LDLT $(n=84)$ for recurrent HCC following curative resections, between January 2005 and December 2017 at a single institution; 1:1 propensity score matching led to 49 patients in each group.

Results: Overall survival (OS) and recurrence-free survival (RFS) were significantly better in the salvage LDLT group than in the repeat LR group (both, $p=0.000$ ). Multivariate analysis revealed that the number of HCCs in explants obtained during salvage LDLT was an independent risk factor for both OS $(p=0.043)$ and RFS $(p=0.014)$.

Conclusions: The results indicate that salvage LDLT is superior to repeat LR for treating patients with transplantable, intrahepatic HCC recurrence, including those with Child-Pugh class A liver cirrhosis. 